\title{
Leveraging Connectivity for Coverage in Drone Networks for Target Detection
}

\author{
E. YANMAZ ADAM
}

\begin{abstract}
Target or event detection is one of the main applications of drone networks. Several cooperative search algorithms have been proposed for teams of unmanned aerial vehicles (UAVs), where the goal is to minimize search time or maximize detection probability. In these works, connectivity often is considered a constraint in enabling cooperation. In this paper, we approach the target detection problem in drone networks from both detection and connectivity viewpoints. Our goal is not only to find a stationary target but also to inform the ground personnel (e.g., a rescue team) about the status of the target over a multi-hop communication chain. We analyze the performance of our coverage-based and connectivity-based path planning algorithms in terms of probability and time of detection as well as notification. We show that there is a trade-off between coverage and connectivity and with limited number of drones both aspects need to be considered for successful mission completion.
\end{abstract}

Index Terms - drones, monitoring, networking, swarms, target detection.

\section{INTRODUCTION}

$\mathrm{D}$ RONE networks are being deployed for environmental monitoring, surveillance, infrastructure inspection, delivery systems, search and rescue to name a few [1]-[4]. For applications such as wildfire tracking, glacier or volcano monitoring, liveliness detection, the area to be monitored can be physically inaccessible or dangerous for humans to enter or the emergency personnel might need to cover the area of interest with high efficiency to be able to respond to the detected event in a timely manner. In such cases, use of drone networks can be extremely beneficial.

In this work, we consider a team of small UAVs (e.g., multirotors) equipped with sensors (e.g., cameras, GPS), network interface (e.g., WiFi), and computation power. The aim of this network is to search a certain area and detect and monitor a target. Only the boundaries of the area the UAV network needs to search is known. We consider two main challenges: i) achieving spatial coverage in an efficient manner (for target

EVŞEN YANMAZ ADAM, Department of Electrical and Electronics Engineering, Faculty of Engineering, Ozyegin University, Istanbul, Turkey, (e-mail: evsen.yanmaz@ozyegin.edu.tr).

iD https://orcid.org/0000-0003-2983-1978 detection) and ii) establishing and maintaining communication links between UAVs and the ground station (for target monitoring). In our previous work, we have shown that there is a trade-off between area coverage and connectivity [5], [6]. For a given number of UAVs, an area of interest can be observed faster if the trajectories of the drones minimally overlap. On the other hand, the farther the drones fly from each other the more difficult it will be to stay connected and exchange observations. In this work, we analyze two path planning models based on potential fields for a team of UAVs, where each UAV autonomously decides its path, taking into account their own observations and position of their immediate neighboring teammates. In particular, the UAVs adapt their directions maintaining either efficient coverage [7] or connectivity [5].

We compare the performance of the coverage- and connectivity-based path models for a UAV network toward a target detection mission. We make no assumptions regarding the sensors onboard the UAVs that will be used for detection, except that the target will be detected with probability 1 if it is within the coverage of at least one UAV; i.e., the detection sensors are considered to be error-free. The mission is considered successful if a time-limited target is found and the ground station is informed about it before the target disappears. We numerically investigate several scenarios, where communication links between the UAVs and the ground station can be formed over a multi-hop network. We analyze the performance in terms of probability of detection and notification as well as mission times. In this work, probability of detection is affected by target duration and not sensing quality. The latter will be analyzed in our future work. Our results show that with the right combination of resources (e.g., number of drones, transmission power) equivalent performances can be achieved from the coverage- and connectivity-based schemes. This indicates that both connectivity and coverage requirements need to be taken into account while planning the paths of UAV networks that are deployed for search applications.

The remainder of the paper is organized as follows. In Section II, related work on networking and path planning in drone networks is provided. The analyzed coverage- and connectivity-based path planning strategies are presented in Section III. Results are given in Section IV and the paper is concluded in Section V. 


\section{RELATED WORK}

\section{A. Wireless Networking with UAVs}

From a networking viewpoint, the multi-UAV system or drone network is classified as flying ad hoc network (FANET) in [8] and differentiated from mobile ad hoc networks (MANET) and vehicular ad hoc networks (VANET) in terms of node mobility and density, frequency of topology change, radio propagation, power consumption, computation power, and localization. A FANET is usually deployed toward a mission, and hence has some common traits with a wireless sensor network as well (see [9], [10], [11]). For instance, sensor node activating strategies are considered in static wireless networks such that coverage time is minimized or detection probability of a target is maximized, or event coverage and connectivity are integrated [9]. Mobile sensor networks have been shown to improve the network capacity and coverage [12], [13]. Mobile robots with swarming capability that operate cooperatively and aim to achieve a global goal have also been considered (see [14], [15], [16]). Meshed communication architecture (multihop) is shown to offer flexibility, performance, and reliability [17]. However, mobility leading to sparsely connected nodes and fast changing network topology is still a key issue to be addressed.

\section{B. Path planning}

From robotic network viewpoint, the team of drones need to be coordinated and we interpret coordination within the scope of our network as multi-uav path planning. Several planning strategies exist for ground robots [18], delivery systems, or mobile sensor networks [14]. Some strategies use prior information with exact or partial decomposition of the areas, whereas others use sensor-based information to make navigation decisions. Path planning and swarming algorithms that rely on network connectivity between drones are also being investigated [5], [19]-[21].

Basic path planning approaches are based on cell decomposition, roadmaps, and potential fields [22], [23]. Cell decomposition methods (exact or approximate) partition the configuration space into a finite number of regions, marking the obstacles on the grid. Roadmaps (such as visibility graphs and Voronoi diagrams) pre-compute a graph such that obstacles can be avoided by staying on the "roads". Approaches based on potential fields on the other hand utilize attractive forces toward the goal and repulsive forces from the obstacles. The use of UAVs has been proposed for target detection applications (see [4] and references therein), where search algorithms have been used for path planning. The target detection algorithms aim to either provide fast area coverage [24], or maximize probability of target detection [25]. Path planning for search usually utilizes communication for information merging to improve the target detection probability [4]. In [7], the transmission ranges are used to create potential fields to repel the mobile nodes in the network to maximize area coverage. However, the successful completion of such missions depend both on locating the target and informing the BS about the target location.

\section{Coverage And ConNectivity Based PATH PlanNing}

In our recent work, we have proposed two path planning models for fast area coverage [7] and for connectivity [5]. Both models make use of the local physical topology information and rely on artificial potential fields to determine the next action of each UAV in a UAV swarm in a distributed manner. The performance of the algorithms will be tested for target detection later in the paper.

\section{A. System Model}

We assume that there is no prior knowledge of the search area accept for its boundaries. The UAVs can have different capabilities, they can enter and leave the system at will. The area of interest can also be dynamic and the proposed algorithms can adapt to it. The UAV team consists of $N_{m}$ drones, each with a sensing range of $r$ and a transmission range of $r_{c}$ (with a disc coverage). The drones fly with speeds uniformly distributed in $\left[0 ; V_{m}\right]$. In this work, we assume the drones all fly at the same altitude $(h)$. The extension of the work for movement in three-dimensional air space is beyond the scope of the paper.

The UAVs know their own positions (e.g., from onboard GPS). They are equipped with sensors (e.g., cameras) that can detect a target as soon as the target is within their coverage. There are no further assumptions regarding the type of detection sensors and the processing time of the sensed information. As a rule, each UAV senses its neighbors every $t_{s}$ seconds and depending on its location at the end of next timestep given its current heading it decides if it needs to change direction. The UAVs exchange only their current location and/or direction. Same algorithm is run on all UAVs and the directions are updated accordingly. If, at the time of direction change, a UAV does not have any neighbors, the direction is not changed. Note that the step length $\left(t_{s}\right)$ is a design parameter and depends on the system parameters such as $N_{m}$ and $r$ among others.

In the following, we briefly explain how coverage- and connectivity-based path planning strategies work. For a detailed analysis of both schemes for area coverage, readers are referred to [5], [7].

\section{B. Coverage-based distributed path planning}

The objective of this path planning strategy is to search a given geographical area by a drone network. Since the goal is fast coverage, the overlap between the covered areas by the limited number of drones need to be minimal. As explained above, there is no centralized controller that optimizes the trajectories of each drone jointly. We assume that each drone decides its own path using the coverage-based path planning algorithm, which changes the direction of the drones at the end of each time step, if necessary. Such a distributed approach is advantageous especially in the case of sparse connectivity between drones and the ground control.

The coverage-based path planning algorithm, which is run on each UAV, is illustrated in Fig. 1. On each UAV $i$, the algorithm takes as input the area of interest $(\mathbf{A})$, sensing period $\left(t_{s}\right)$, transmission range $(r)$, current direction and position of node $i\left(\theta_{i}{ }^{c}, P_{i}\right)$, and position of neighboring drones $\left(\left\{N_{i}\right\}\right)$. It returns the next direction $\left(\theta_{i}^{+}\right)$. 
The main direction decision depends on a drone's current position and direction relative to the position of its neighboring teammates and it happens every $t_{s} \sec$ (update time). Timer starts at zero and is increased at increments much smaller than $t_{s}$. At each time increment $t_{i}$, the direction of a drone can change if the drone notices it would leave the search area $\mathbf{A}$, based on its estimated position $\left(P_{i}^{+}\right)$given its current direction. In that case, the drone chooses a random direction $\left(\theta_{i}^{+}\right)$toward $\mathbf{A}$. Once the total time increment reaches $t_{s}$, each drone computes the resultant force $\left(\vec{R}_{w}\right)$ acting on it and the timer is set to zero. In particular, each drone pushes its neighboring drones with a force $\left(\vec{F}_{i i}\right)$ that is inversely proportional to the distance between the nodes. An attraction force $\left(\vec{F}_{i i}\right)$ which is inversely proportional to the drone's sensing range (i.e., $r$ ) is also applied to avoid backtracking. Each drone, then, moves in the direction of the resultant vector, which is the sum of all applied forces on the drone of interest. The calculation of the applied forces and the resultant force is shown in Fig. 1, where $\Theta_{i}{ }^{c}$ and $\Theta_{i j}$ are the unit vectors with angles $\theta_{i}{ }^{c}$ and $\theta_{i j}$, respectively.

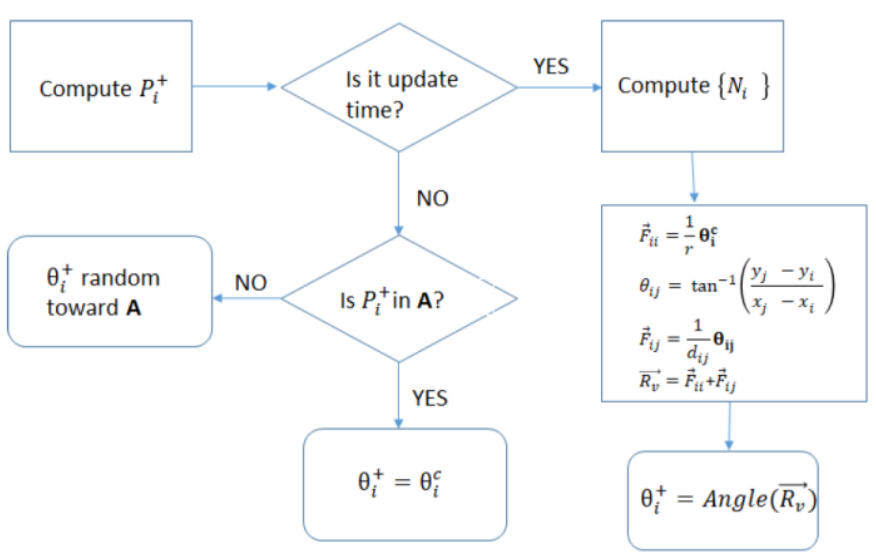

Fig.1. Coverage-based path planning algorithm

\section{Connectivity-based distributed path planning}

This path planning strategy takes communication requirements into account. The goal of the drone network is to search a given area, while the drones maintain connectivity to the BS (sink node) and/or their neighbors. Due to the probabilistic nature of the algorithm, disconnections from the BS can occur, but likelihood of isolated drones is low. The parameters used in the algorithm running on each UAV are defined in Table I and the algorithm is illustrated in Fig. 2. We denote the UAV of interest as UAV $i$.

As with the previous strategy, connectivity-based path planning is also distributed and every $t_{s}$ seconds, each UAV computes their next direction. Similarly, at each time increment $t_{i}$ during a timestep of $t_{s}$, the drones ensure they do not leave $\mathbf{A}$.
TABLE I

ALGORITHM PARAMETERS

\begin{tabular}{|l|l|}
\hline Parameter & Definition \\
\hline$t_{s}$ & sensing period \\
\hline$t_{i}$ & time increment \\
\hline$r_{c}$ & transmission range \\
\hline $\boldsymbol{A}$ & search area \\
\hline$A_{s}$ & coverage area of BS \\
\hline$\theta_{i}{ }^{c}$ & current direction of drone $i$ \\
\hline$\theta_{i}{ }^{+}$ & next direction of drone $i$ \\
\hline$P_{i}$ & current position of drone $i$ given by $\left(x_{i}, y_{i}, h\right)$ \\
\hline$P_{i}{ }^{+}$ & position of drone $i$ given by $\left(x_{i}{ }^{+}, y_{i}{ }^{+}, h\right)$ after $t_{i}$ sec \\
\hline$P_{i}^{\text {next }}$ & position of drone $i$ given by $\left(x_{i}^{\text {next }}, y_{i}^{\text {next }}, h\right)$ after $t_{s}$ sec \\
\hline$\left\{N_{i}\right\}$ & $\begin{array}{l}\text { current positions of set of neighbors of drone } i \text { given by } \\
\left(x_{j}, y_{j}, h\right)_{i}\end{array}$ \\
\hline$\left\{N_{i}{ }^{+}\right\}$ & $\begin{array}{l}\text { positions of current neighbors of drone } i \text { after } t_{i} \text { sec } \\
\text { given by }\left(x_{j}{ }^{+}, y_{j}{ }^{+}, h\right)_{i}\end{array}$ \\
\hline$\left\{N_{i}{ }^{\text {next }}\right\}$ & $\begin{array}{l}\text { positions of current neighbors of drone } i \text { after } t_{s} \text { sec } \\
\text { given by }\left(x_{j}^{\text {next }}, y_{j}^{\text {next }}, h\right)_{i}\end{array}$ \\
\hline$R^{+}$ & network routing table for a given $P_{i}{ }^{+},\left\{N_{i}{ }^{+}\right\}$ \\
\hline$R^{\text {next }}$ & network routing table for a given $P_{i}^{\text {next }},\left\{N_{i}{ }^{\text {next }}\right\}$ \\
\hline
\end{tabular}

Different than coverage-based algorithm, at each increment drone $i$ computes its routing table at the end of $t_{i}$ and $t_{s}$, assuming there would be no direction change. Using these routing table estimates, drone $i$ checks whether it would be connected to the BS at the end of $t_{s}$. If yes, it continues with its current direction $\theta_{i}{ }^{c}$.

If not, the drone $i$ aims to avoid being isolated by staying connected to the BS or one of its neighbors which we will denote by drone $j$. First, each drone checks whether the BS is an immediate neighbor. If it is, the drone chooses a random direction toward the BS coverage area $\left(A_{s}\right)$ to ensure connectivity. If the next hop in the route is not the BS, we have two cases. Algorithm checks $R^{+}$to see if the UAV $i$ has a route to the BS at the end of a time increment even if it doesn't have a route at the end of $t_{s}$. If it has a route, then $j$ is set to be the next hop neighbor of $i$ in the multi-hop route to BS. If it is not connected to the BS after $t_{i}$ either, drone $i$ calls the function max_contact_id. This function estimates the contact time to each neighbor (i.e., the time UAV $i$ would be connected to a given neighbor if all neighbor UAVs in $\left\{N_{i}{ }^{+}\right\}$ keep their current heading) and chooses the neighbor with longest estimated contact time. Parameter $j$ in this case is set to the ID of this neighboring node. All of these steps are taken to keep the network connected while avoiding frequent direction changes.

Once the node $j$, to which the UAV $i$ decides to stay connected, is determined, we again use artificial potential fields to determine the next direction $\theta_{i}{ }^{+}$. The forces that apply on drone $i$ are 1) a force that pulls the UAV in its current direction $\left(\vec{F}_{i i}\right)$ with unit magnitude and 2 ) a force that pulls the UAV toward the last contact point with neighbor $j$, should both UAVs keep their direction $\left(\vec{F}_{i j}\right.$, again with unit magnitude). This way UAV $i$ is gradually pulled toward neighbor $j$ while keeping the overlap between coverages still small. Recall that the goal is to search an area to find targets as fast as possible. As such, we want to keep the drones as far from each other as possible, while still being connected. Exact 


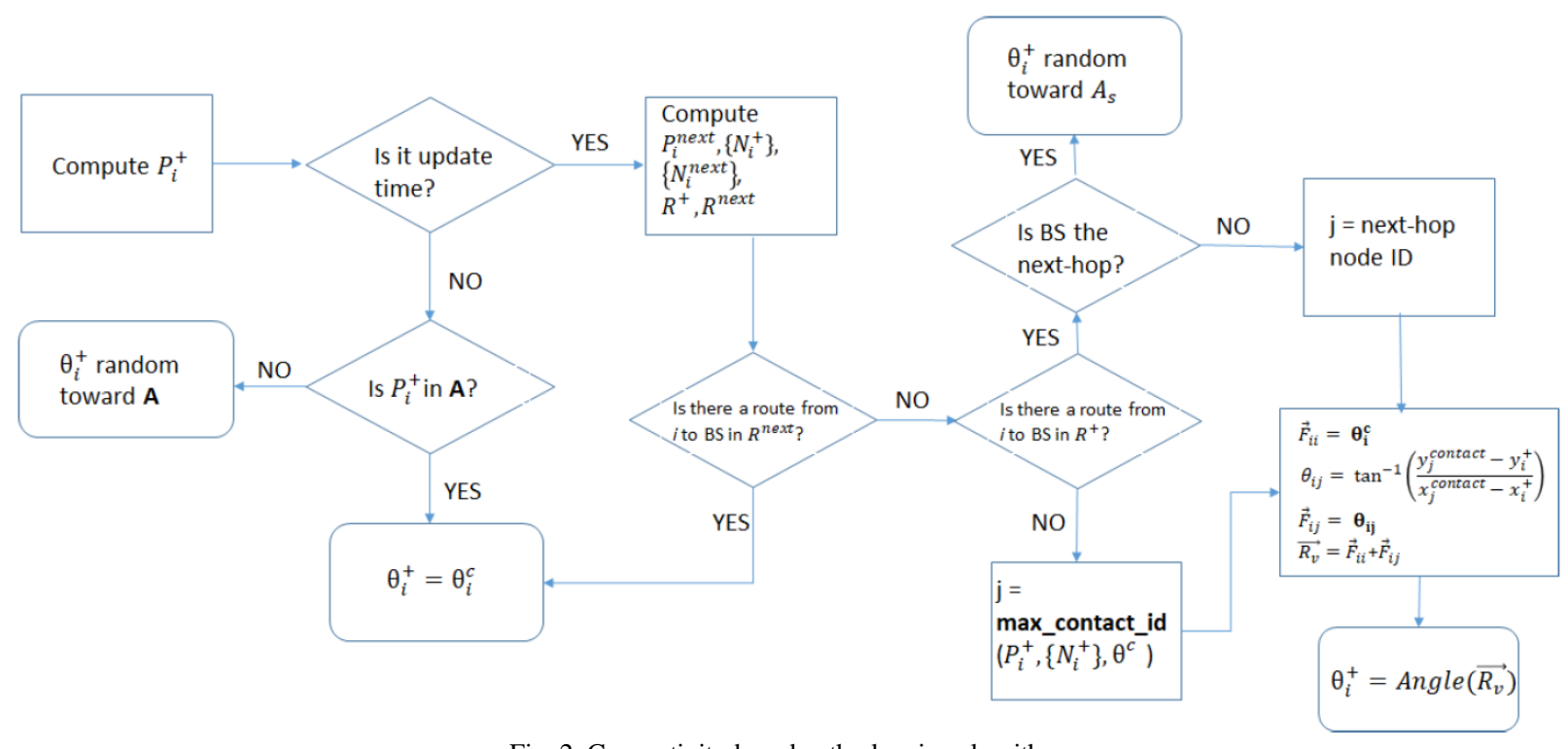

Fig. 2. Connectivity-based path planning algorithm.

calculation of the applied forces and resultant force is given in Fig. 2.

If a UAV nevertheless becomes isolated (i.e., because $t_{s}$ is too long, the transmission range of the sink is too short, or the UAV speed is too high), it does not change its direction until it reaches the boundary or it meets another UAV.

\section{RESUlTS AND DiscUSSION}

In this section, we compare the target detection and BS notification performance of the coverage and connectivitybased path planning strategies via Monte Carlo simulations. Each data point is computed over 2000 different runs. The simulation area is square-shaped with a length of $4000 \mathrm{~m}$. The UAVs start their mission above the ground station, which is placed at center of the search area. The travel time of the UAVs is assumed to be $2500 \mathrm{~s}$ and their velocity is fixed to 5 $\mathrm{m} / \mathrm{s}$. The sensing range, $r$, of the UAVs is set to $500 \mathrm{~m}$ and the sensing period for direction change is $2 \mathrm{~s}$. We study a multihop system, where the UAVs are connected to the ground station via a shortest-hop network route. The number of hops in the routes between the UAVs and the ground station depend on the transmission range $r_{c}$ of the nodes.

We assume that a single event occurs at a random location within the simulation area and lasts for a duration of $t_{d}$ seconds. $N_{m}$ drones search the area. Assuming a target is detected without error as soon as it is within sensing range of a UAV, the performance metrics of interest are: i) probability of detection within $t_{d}$; ii) time to detect; iii) probability of immediate notification (at the time of detection); iv) probability of later notification (within $t_{d}$ ); v) time to notify; and v) total mission time, where the mission is over once the BS is notified (i.e., includes search time and notification time).

We also provide the detection probability of an ideal mobile network, where there is minimal to no overlap between drone trajectories. In [7], we have shown that the percentage of covered area by at least one node in $[0 ; t]$ is:

$$
P_{c_{m}}=1-e^{-N_{m}\left(\pi r^{2}+2 r E[V] t\right) / A}
$$

where $r$ is the disc sensing range of the drones, $N_{m}$ is the number of drones, $V$ is the uniformly distributed velocity within $\left[0 ; V_{m}\right]$, and $A$ is the total area to be covered.

\section{A. Detection performance}

First, we study the impact of target duration and number of drones on the probability of detection performance of coverage- and connectivity-based path models. Fig. 3 shows the probability of detection versus target duration, when $N_{m}=$ $\{6,26\}$ and $r_{c}=\{500,1000,2000\} \mathrm{m}$. Analytical results for mobile and static random network are obtained using Equation(1). Observe that coverage-based paths perform as well as the ideal mobile network if $t_{d}$ is long enough for both $N_{m}$ values. The need for static nodes can be very high, illustrating that mobility improves coverage. The probability of detection for both schemes increase with increasing $t_{d}$, eventually becoming 1 . The performance of connectivitybased mobility model depends on the transmission range. If $r_{c}$ is large enough the drones can freely spread in the search area, bringing the performance of connectivity-based scheme closer to that of coverage-based scheme. If there are enough number of nodes, the performance of the two schemes become equivalent, since the drone network eventually becomes connected due to increased spatial node density.

Fig. 4 shows the probability of detection versus $N_{m}$, when target duration is $\{300,2400\} \mathrm{s}$. Similar to the previous case, coverage-based mobility model performs better if $r_{c}$ is not large enough. While for short target durations, the two schemes perform worse than an ideal mobile network, the benefit of mobility over static network can be observed even with short target durations, when the number of drones is low. If the target duration is short, adding more drones into the 


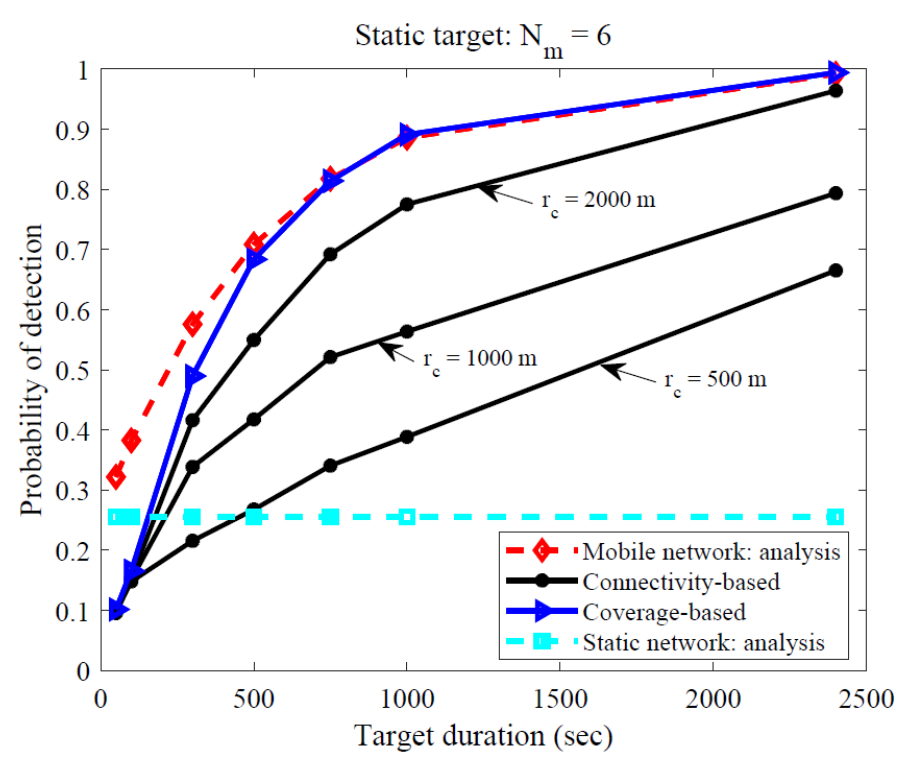

(a) $N_{m}=6$

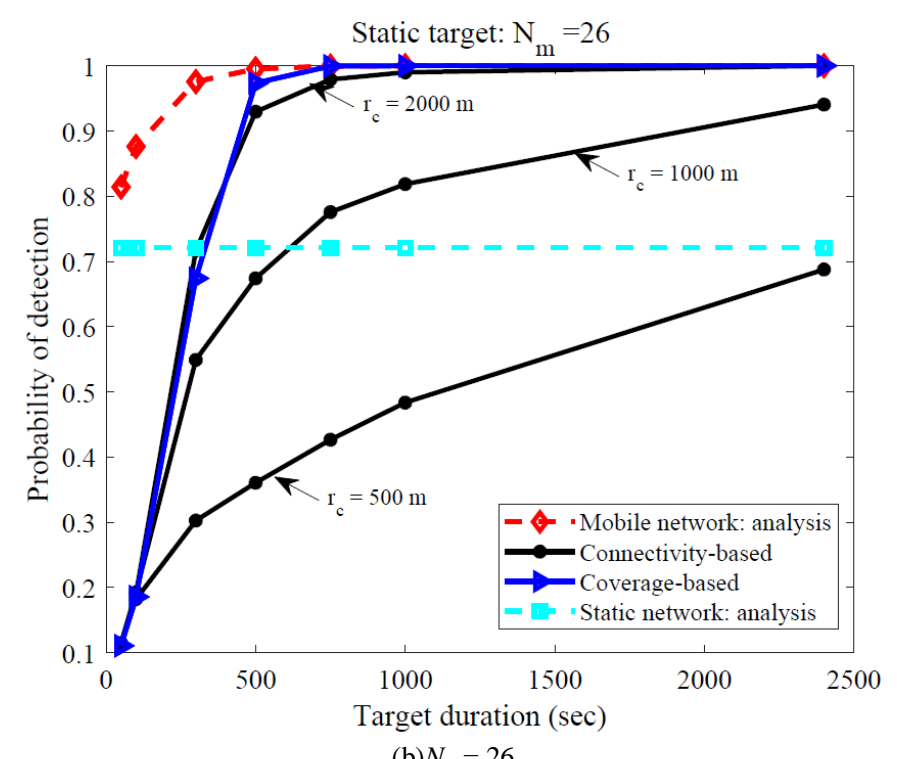

(b) $N_{m}=26$

Fig. 3. Probability of detection versus target duration

network does not improve the performance, since all nodes depart from a single ground station. If the target duration is long, on the other hand, even with a low number of drones the target can be detected as expected. If the transmission range is low (e.g., $r_{c}=500 \mathrm{~m}$ ), the performance of the connectivitybased scheme is limited and a much higher number of drones would be required to achieve an acceptable performance.

\section{B. Notification performance}

In the previous section, we have seen that the detection performance of the connectivity-based path model is limited by the transmission range. In the following, we will illustrate that conversely the notification performance of the coveragebased model is limited by the transmission range. The probability of notification is conditioned on detection. Fig. 5

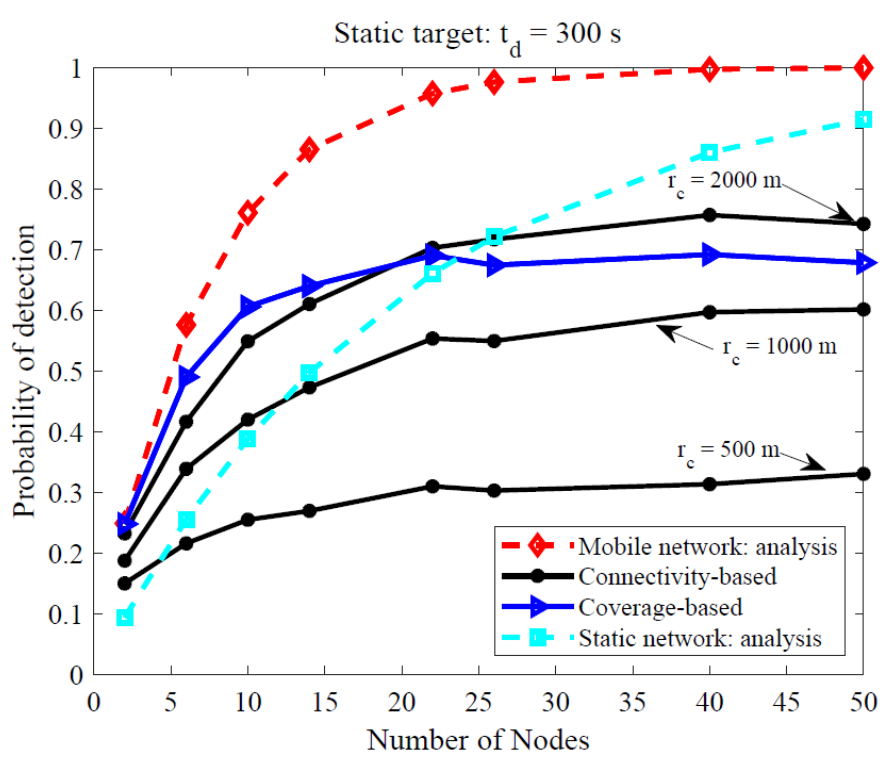

(a) $t_{d}=300 \mathrm{~s}$

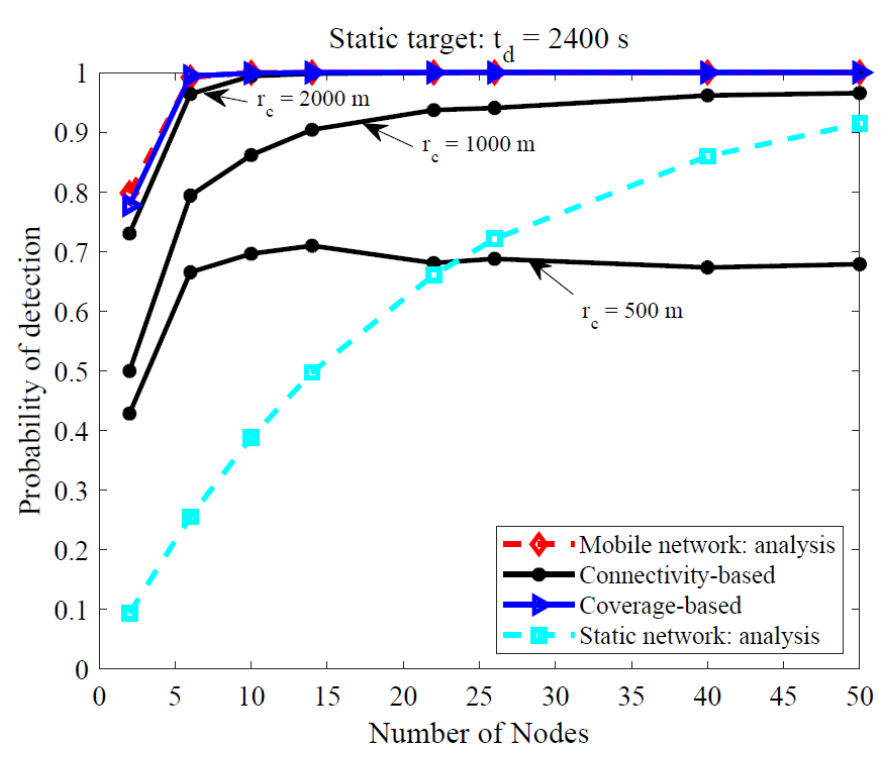

(b) $t_{d}=2400 \mathrm{~s}$

Fig. 4. Probability of detection versus number of nodes.

shows the probability of notification performance versus $N_{m}$, when target duration is $2400 \mathrm{~s}$. We analyze the immediate notification probability (a) as well as notification probability before the target disappears (b). Fig. 5 (c) shows the total notification probability. We also provide the simulation results for a random static network, where the total notification probability is the same as the immediate notification probability. We observe that since the connectivity-based scheme takes into account the transmission range of the drones when determining the drone movements, the drones stay connected throughout the mission with a high probability. Therefore, the likelihood that the BS will immediately be notified is very high and when $r_{c}$ becomes high enough (1000m in the figure), the likelihood becomes one even with low number of nodes. Even for low transmission ranges, the 
performance of connectivity-based scheme is more than twice of the coverage-based scheme when $N_{m}$ is low. When $r_{c}$ is $1000 \mathrm{~m}$, coverage-based scheme needs 40 drones for an immediate notification probability of 1 , whereas connectivitybased scheme needs around 15. Furthermore, with 15 drones coverage-based scheme could detect the target with probability 1 (Fig. 4), whereas connectivity-based scheme would detect with a probability of 0.9 . Therefore, the gain in immediate notification probability for connectivity-based model is more prominent than the gain in detection probability for coverage-based model.

Conversely, probability of later notification is much higher for coverage-based model. If the eventual notification of the BS within the target duration is sufficient, immediate notification might not be essential. Observe from Fig. 5 (c) that the total notification performance of the two schemes is very similar if the number of drones is high enough.

\section{Mission time performance}

So far, we have looked on the detection and notification performance in terms of probabilities. It is important to determine relative times for different parts of the mission and also how late is "later notification". Fig. 6 shows the time required to detect the target (a) and notify the BS (b), as well as the total mission time that includes both the detection and notification phases. As expected, the time to detect is shorter for coverage-based scheme and the time to notify is shorter for the connectivity-based scheme. The difference reduces as the transmission range and the number of drones (i.e., the spatial node density) increases. In terms of the total mission time, the connectivity-based scheme outperforms the coverage-based scheme even with low transmission ranges, when $t_{d}$ is high. This, however, should be considered together with the fact the mission success probability can be less for connectivity-based schemes when the target duration is low (recall the probability of detection when $t_{d}=300 \mathrm{~s}$ ), especially when $r_{c}$ is low.

\section{CONCLUSIONS}

In this work, local, cooperative distributed path planning methods for a UAV team are analyzed for a target detection application in terms of detection and connectivity performance. We analyze two of our models: coverage-based and connectivity-based. As expected, the path decisions of the UAVs for the two schemes have conflicting goals. Whereas the coverage-based model aim to spread the UAVs as much and fast as possible, the connectivity-based model aims to keep the team together so that information on the target can immediately be exchanged. The performance of the models in terms of detection and notification reflects this conflict. When the number or the transmission range of drones increase the performance difference between the two schemes reduce. However, the available resources depend on the cost and goal of a mission. Therefore, we conclude that the choice of the path planning model depends on the needs of the mission. With the right combination of resources (e.g., number of drones, transmission power) equivalent performances can be achieved. A potential approach could utilize the coverage and connectivity benefits of the two schemes and adapt to the mission needs. A weighted approach that combine the two schemes is currently under investigation.

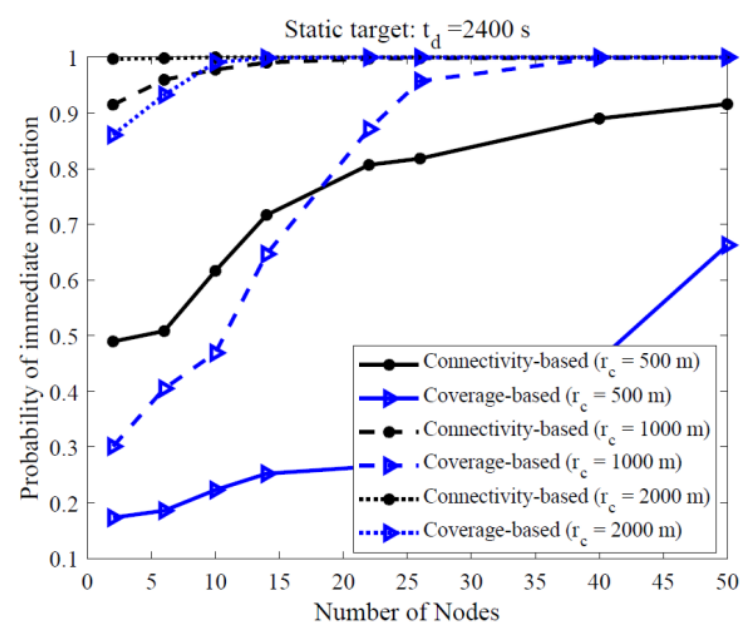

(a)Probability of immediate notification

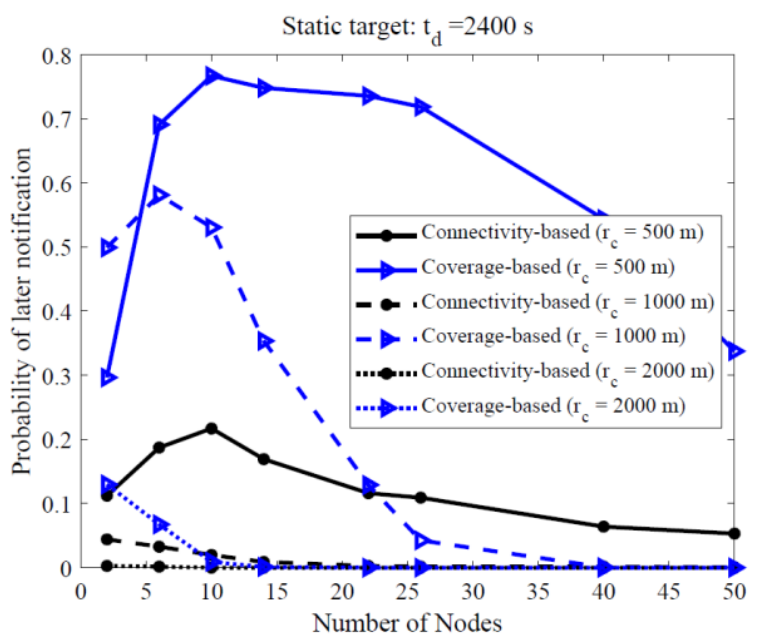

(b)Probability of later notification

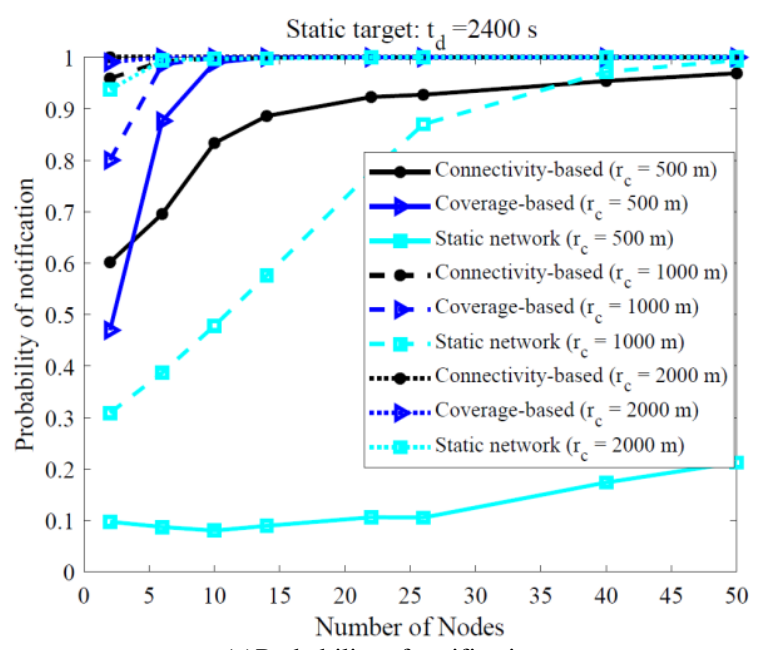

(c)Probability of notification

Fig. 5. Probability of notification versus number of nodes when $t_{d}=2400 \mathrm{~s}$. 


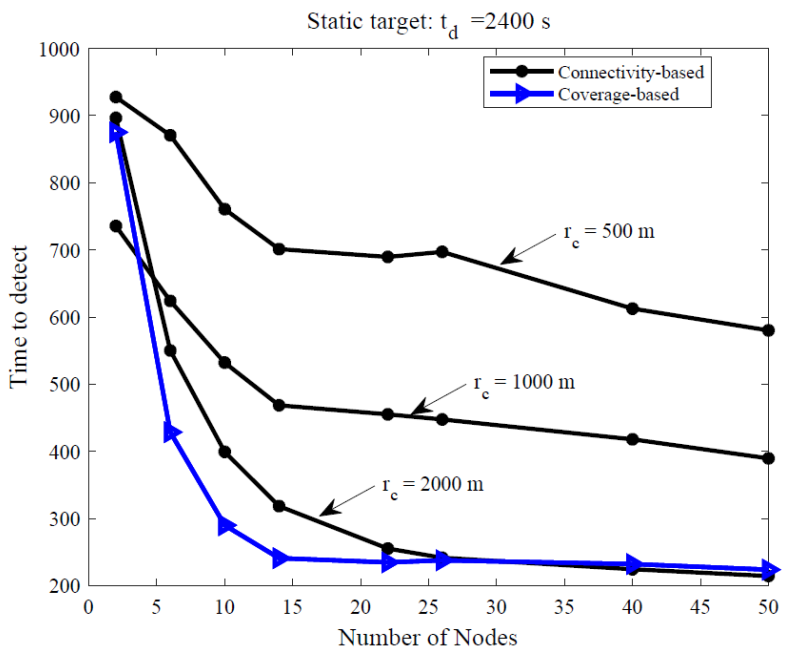

(a) Detection time

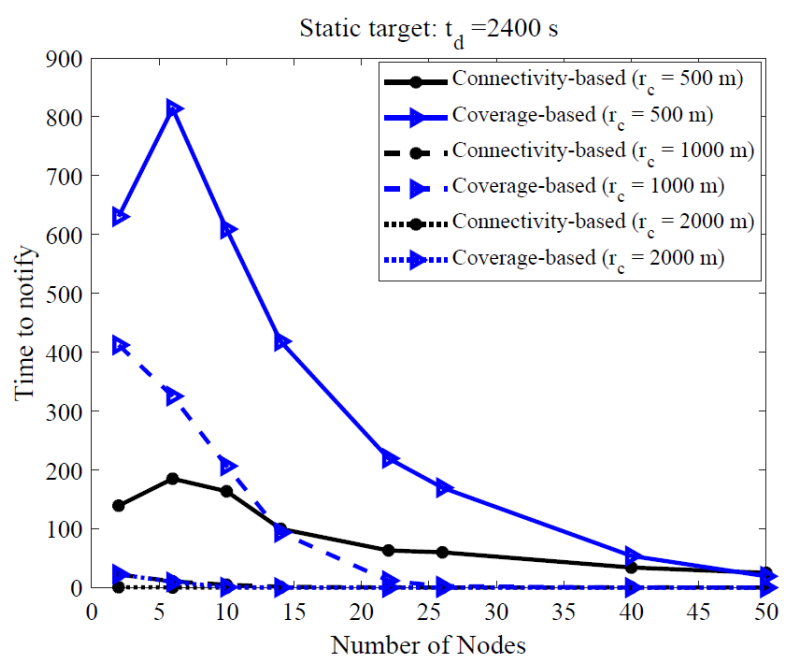

(b) Notification time

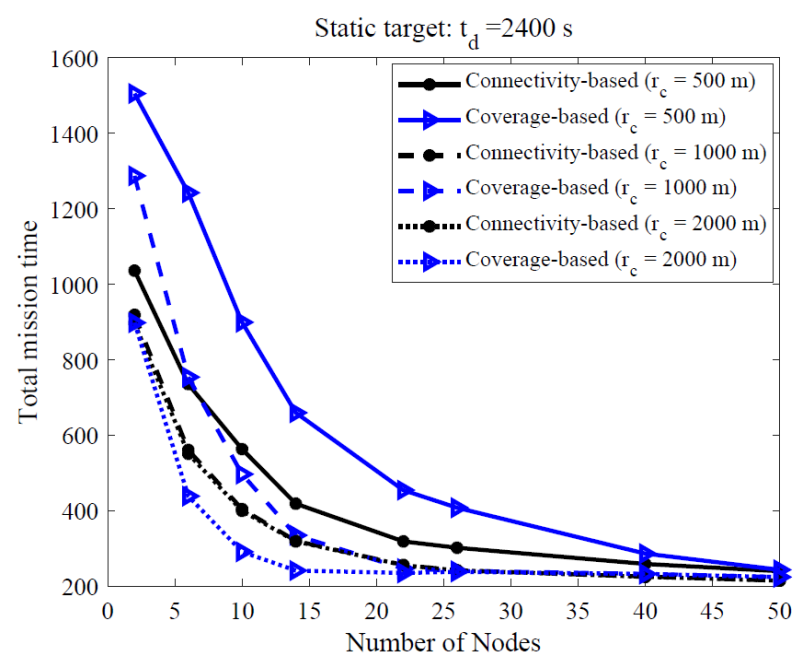

(c) Total mission time

Fig. 6. Mission phase times versus number of nodes when $t_{d}=2400 \mathrm{~s}$.

\section{REFERENCES}

[1] D. Cole, A. Goktogan, P. Thompson, and S. Sukkarieh, "Mapping and tracking," IEEE Robotics Automation Magazine, vol. 16, no. 2, pp. $22-34$, June 2009.

[2] S. Hayat, E. Yanmaz, and R. Muzaffar, "Survey on unmanned aerial vehicle networks for civil applications: A communications viewpoint," IEEE Communications Surveys Tutorials, vol. 18, no. 4, pp. 2624-2661, Fourthquarter 2016.

[3] M. Erdelj, E. Natalizio, K. R. Chowdhury, and I. F. Akyildiz, "Help from the sky: Leveraging UAVs for disaster management," IEEE Pervasive Computing, vol. 16, no. 1, pp. 24-32, Jan 2017.

[4] A. Khan, E. Yanmaz, and B. Rinner, "Information Exchange and Decision Making in Micro Aerial Vehicle Networks for Cooperative Search," IEEE Transactions on Control of Network Systems, vol. 2, no. 4, pp. 335-347, 2015.

[5] E. Yanmaz, "Connectivity versus area coverage in unmanned aerial vehicle networks," in Proc. IEEE Int. Conf. on Communications (ICC), 2012. [6] S. Hayat, E. Yanmaz, T. Brown, and C. Bettstetter, "Multi-objective UAV path planning for search and rescue," in Proc. Intl. Conf. Robotics and Automation, ser. ICRA '17, 2017.

[7] E. Yanmaz and H. Guclu, "Stationary and mobile target detection using mobile wireless sensor networks," in Proc. IEEE Conf. on Computer Communications (INFOCOM), Mar. 2010, pp. $1-5$.

[8] I. Bekmezci, O. K. Sahingoz, and C. Temel, "Flying Ad-Hoc Networks (FANETs): A Survey," Ad Hoc Networks, vol. 11, no. 3, pp. 1254-1270, May. 2013.

[9] X. Wang, G. Xing, Y. Zhang, C. Lu, R. Pless, and C. Gill, "Integrated coverage and connectivity configuration in wireless sensor networks," in Proc. Int's. Conf. Emb. Net. Sens. Sys. (SenSys'03), 2003, pp. 28-39.

[10] B. Liu and D. Towsley, "A study of the coverage of large-scale sensor networks," in Proc. IEEE Int'l. Conf. Mob. Ad hoc Sens. Sys. (IEEE MASS'04), Oct. 2004, pp. 475-483.

[11] S. Megerian, F. Koushanfar, M. Potkonjak, and M. B. Srivastava, "Worst and best-case coverage in sensor networks," IEEE Trans. Mob. Comp., vol. 4, no. 1, pp. 84-92, Jan./Feb. 2005.

[12] M. Grossglauser and D. N. C. Tse, "Mobility increases the capacity of ad hoc wireless networks," IEEE/ACM Trans. Networking, vol. 10, no. 4, pp. 477-486, Aug. 2002.

[13] B. Liu, P. Brass, O. Dousse, P. Nain, and D. Towsley, "Mobility improves coverage of sensor networks," in Proc. ACM Intl. Symp. Mobile Ad hoc Networking and Computing (MobiHoc '05), 2005, pp. 300-308.

[14] S. Poduri and G. S. Sukhatme, "Constrained coverage for mobile sensor networks," in Proc. IEEE Intl. Conf. on Robotics and Automation, 2004, pp. $165-172$.

[15] P. Vincent and I. Rubin, "A framework and analysis for cooperative search using UAV swarms," in Proc. ACM Symp. Applied Computing, 2004, pp. 79-86.

[16] Y. Jin, Y. Liao, M. M. Polycarpou, and A. A. Minai, "Balancing search and target response in cooperative unmanned vehicle teams," IEEE Trans. on Sys., Man and Cybernetics, vol. 36, pp. 571-587, 2006.

[17] E. W. Frew and T. X. Brown, "Airborne communication networks for small unmanned aircraft systems," Proc. of the IEEE, vol. 96, no. 12, 2008.

[18] H. Choset, "Coverage for robotics - a survey of recent results," Annals of Math. and Artificial Intel., vol. 31, no. 1-4, pp. 113-126, 2001.

[19] S. Hauert, S. Leven, J.-C. Zufferey, and D. Floreano, "Communicationbased swarming for flying robots," in Proc. Intl. Conf. Robotics and Automation Workshop on Network Science and Systems, 2010.

[20] Y. Mostofi, "Communication-aware motion planning in fading environments," in Proc. Intl. Conf. Robotics and Automation, 2008, pp. 31693174.

[21] E. F. Flushing, M. Kudelski, L. M. Gambardella, and G. A. D. Caro, "Connectivity-aware planning of search and rescue missions," in Proc. IEEE International Symposium on Safety, Security, and Rescue Robotics (SSRR), Oct 2013, pp. 1-8.

[22] E. Frazzoli, M. Dahleh, and E. Feron, "Real-time motion planning for agile autonomous vehicles," Journal of Guidance, Control, and Dynamics, vol. 25, pp. 116-129, 2002.

[23] J.-C. Latombe, Robot Motion Planning. Kluwer, 1991.

[24] S. Waharte and N. Trigoni, "Supporting search and rescue operations with UAVs," in Proc. International Conference on Emerging Security Technologies (EST), October 2010, pp. 142-147. 
[25] L. Lin and M. A. Goodrich, "UAV intelligent path planning for wilderness search and rescue," in Proc. IEEE/RSJ International Conference on Intelligent Robots and Systems (IROS), Oct 2009, pp. 709-714.

\section{BIOGRAPHIES}

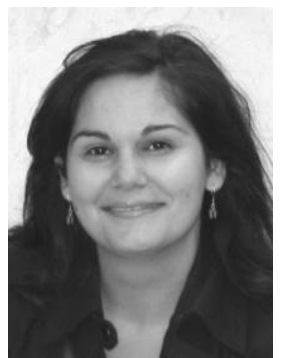

EVSEN YANMAZ received the BS degree in electrical and electronics engineering from Bogazici University in 2000, the MS degree in electrical engineering from SUNY at Buffalo in 2002, and the PhD degree in electrical and computer engineering from Carnegie Mellon University, in 2005. She is an assistant professor at Ozyegin University. Previously, she held positions as a senior researcher and project leader at Lakeside Labs, Klagenfurt, as a senior researcher at the NES Institute at the University of Klagenfurt, as a postdoctoral researcher at the Los Alamos National Laboratory, and as a researcher at Carnegie Mellon University. Her research interests include drone networks, self-organizing networks, cooperative networks, and coordination of airborne and ground sensor networks. She published more than 50 peer-reviewed works focusing on the fields of telecommunications, networking, self-organization, and path planning. 